\title{
POINCARÉ DUALITY AND POSTNIKOV FACTORS
}

\author{
J. W. VICK
}

With the advent of generalized homology and cohomology theories during the last decade there has been a natural movement toward recasting classical results from ordinary homology into these new theories. One of the earliest of the generalizations was the Poincare duality theorem [10]. Perhaps the most interesting problem in this area has been the characterization of the class of orientable manifolds corresponding to each new theory $([6],[7],[9]$ and $[10])$. For example a manifold is orientable for stable homotopy if it is stably parallelizable, for real $\boldsymbol{K}$-theory if it admits a Spin structure and for complex $K$-theory if it admits a $\operatorname{Spin}^{c}$ structure.

In [7] Kan and Whitehead asked if it is possible to fill the gap between the Eilenberg-Mac Lane spectrum $K(Z)$ (integral homology) and the sphere spectrum $S$ (stable homotopy) with interesting spectra and corresponding classes of orientable manifolds. Using semisimplicial methods, they constructed a sequence of ring spectra for which orientability was characterized by the vanishing of certain higher order ordinary homology operations on the integral fundamental class.

Our purpose here is to give an alternate, but related, solution to this problem and in the process to present an approach which should shed light on the orientability question in general. The basic tool is the Atiyah-Hirzebruch-Dold spectral sequence ([2]-[5]). Starting with an axiomatic slant product, it is shown that a corresponding product is induced on the associated spectral sequences which satisfies a derivation property with respect to the differentials $(\$ 2)$. This allows us to proceed from ordinary Poincare duality at the $E^{2}$-stage to prove the generalized theorem at the $E^{\infty}$-stage. A natural result of this approach is that the Poincare duality isomorphism is seen to be an isomorphism of filtered groups, under the natural CW filtrations (\$3).

This leads to the characterization of orientability as the requirement that the fundamental class at the $E^{2}$-stage be a permanent cycle. Then one might ask if there are intermediate homology theories for which a manifold is orientable if and only if its ordinary fundamental class remains a cycle until the $E^{k}$-stage. This question is answered

Received by the editors August 10, 1971 and, in revised form, December 6, 1971 .

AMS (MOS) subject classifications (1970). Primary 55B20, 55B45, 55C05, 55H25; Secondary 57B10, 55J30. 
affirmatively by exhibiting the relationship between the Postnikov factors of the homology theory [4] and the various stages of the spectral sequence. These results are summarized in a Poincare duality theorem for Postnikov factors $(\$ 4)$.

The Postnikov factors of stable homotopy are presented as a solution to the question of Kan and Whitehead ( $\$ 5)$. One reason this alternate solution is interesting is that orientability for the intermediate theories may be given a concise geometrical characterization. Results of Milnor and Spanier [8] show that a manifold is orientable for stable homotopy if and only if its stable normal sphere bundle is fibre homotopically trivial. These techniques may be applied to show that a manifold is orientable for a Postnikov factor if and only if the restriction of the stable normal sphere bundle to the corresponding skeleton is fibre homotopically trivial. Finally, examples are given to show that these theories are nonvacuous $(\$ 6)$.

Much appreciation is due Professor E. E. Floyd for originally suggesting the spectral sequence approach and for many enlightening conversations.

1. Preliminaries. Let $h_{*}$ be a generalized homology theory in the sense of Dold [5]. Thus $h_{*}$ satisfies excision in the sense that if $A$ and $B$ are subcomplexes of a finite CW complex $X$, the homomorphism

$$
i_{*}: h_{*}(B, B \cap A) \rightarrow h_{*}(A \cup B, A)
$$

induced by inclusion is an isomorphism. A generalized cohomology theory is the contravariant analog.

Given finite $\mathrm{CW}$ triples $(C, X, A)$ and $(Y, B, D)$, define a homomorphism

$$
D_{1}: h_{n+1}((C, X) \times(Y, B)) \rightarrow h_{n}((X, A) \times(Y, B))
$$

by the composition $i_{*}{ }^{-1} \circ \partial$ in

$$
h_{n+1}(C \times Y, C \times B \cup X \times Y) \stackrel{\partial}{\rightarrow} h_{n}(C \times B \cup X \times Y, C \times B \cup A \times Y)
$$

$$
\approx i_{*}
$$

$$
h_{n}(X \times Y, X \times B \cup A \times Y)
$$

where $i_{*}$ is an excision isomorphism. Similarly define

$$
D_{2}: h_{n}((X, A) \times(Y, B)) \rightarrow h_{n-1}((X, A) \times(B, D)) .
$$


Now suppose $h^{*}, h_{*}$ and $k_{*}$ are generalized theories. A slant product is a bilinear pairing

$$
\text { I: } h^{p}(X, A) \otimes k_{n}((X, A) \times(Y, B)) \rightarrow h_{n-p}(Y, B)
$$

defined for any finite CW pairs $(X, A)$ and $(Y, B)$ satisfying the following axioms: given maps $f:\left(X^{\prime}, A^{\prime}\right) \rightarrow(X, A)$ and $g:\left(Y^{\prime}, B^{\prime}\right) \rightarrow$ $(Y, B)$ and elements $a \in h^{p}(X, A), b \in k_{n}\left(\left(X^{\prime}, A^{\prime}\right) \times\left(Y^{\prime}, B^{\prime}\right)\right)$ and $c \in k_{n+1}((C, X) \times(Y, B))$ then

(1.1) $a /(f \times g)_{*}(b)=g_{*}\left(f^{*}(a) / b\right)$,

(1.2) $\delta a / c=a / D_{1}(c)$,

(1.3) $a / D_{2}(c)=(-1)^{p} \partial(a / c)$,

where $\partial$ and $\delta$ are the ordinary boundary and cobounding operators of the respective triples.

Examples. (i) There is the usual slant product in ordinary homology and cohomology induced by a pairing of the coefficient groups.

(ii) If $\pi_{*}$ and $\pi^{*}$ are stable homotopy and cohomotopy theory, there is a slant product

$$
\pi^{p}(X, A) \otimes \pi_{n}((X, A) \times(Y, B)) \rightarrow \pi_{n-p}(Y, B) .
$$

To see how this is defined, let $a \in \pi^{p}(X, A)$ be represented by a map $\alpha: S^{k} \wedge(X / A) \rightarrow S^{k+p}$ and $b \in \pi_{n}((X, A) \times(Y, B))$ be represented by a map $\beta: S^{k+n} \rightarrow S^{k} \wedge(X / A) \wedge(Y / B)$, where $k$ is large. Then $a / b \in \pi_{n-p}(Y, B)$ is represented by the composition

$$
\mathrm{S}^{k+n} \stackrel{\beta}{\rightarrow} S^{k} \wedge(\mathrm{X} / \mathrm{A}) \wedge(\mathrm{Y} / \mathrm{B}) \stackrel{\alpha \wedge i d}{\longrightarrow} S^{k+p} \wedge(\mathrm{Y} / \mathrm{B}) .
$$

(iii) In the manner of Whitehead [10] any pairing of spectra gives rise to a slant product on the associated theories. (In fact, this is how examples (i) and (ii) both arise.)

There is a standard spectral sequence discussed by Dold [5], Atiyah and Hirzebruch [2] and others which we now describe. In order to hold notational confusion to a minimum, we deal with single spaces rather than pairs.

Denote by $X^{n}$ the $p$-skeleton of a finite CW complex $X$ and let $h_{*}$ be a generalized homology theory.

Define $E_{p, q}^{1}=h_{p+q}\left(X^{p}, X^{p-1}\right)$ and let $d^{1}: E_{p, q}^{1} \rightarrow E_{p-1, q}^{1}$ be the boundary operator of the corresponding triple. In general the definitions are given by

$$
\begin{aligned}
& Z_{p, q}^{r}=\text { Image }\left[h_{p+q}\left(X^{p}, X^{p-r}\right) \rightarrow h_{p+q}\left(X^{p}, X^{p-1}\right)\right], \\
& B_{p, q}^{r}=\text { Image }\left[h_{p+q+1}\left(X^{p+r-1}, X^{p}\right) \stackrel{\partial}{\rightarrow} h_{p+q}\left(X^{p}, X^{p-1}\right)\right],
\end{aligned}
$$


and $E_{p, q}^{r}=Z_{p, q}^{r} / B_{p, q}^{r}$. The composition $\partial \circ \beta_{*}^{-1}$ given by

$$
h_{p+q}\left(X^{p}, X^{p-1}\right) \stackrel{\boldsymbol{\beta}^{*}}{\longleftarrow} h_{p+q}\left(X^{p}, X^{p-r}\right) \stackrel{\partial}{\rightarrow} h_{p+q-1}\left(X^{p-r}, X^{p-r-1}\right)
$$

induces the differential $d^{r}: E_{p, q}^{r} \rightarrow E_{p-r, q+r-1}^{r}$.

If $\operatorname{dim} X=n$, there is filtration

$$
0=J_{-1, m+1} \subseteq J_{0, m} \subseteq \cdots \subseteq J_{n, m-n}=h_{m}(X)
$$

where

$$
J_{p, q}=\operatorname{Image}\left[h_{p+q}\left(X^{p}\right) \rightarrow h_{p+q}(X)\right] \quad \text { and } \quad J_{p, q} / J_{p-1, q+1} \approx E_{p, q}^{\infty} .
$$

Finally there is the identification [5]

$$
E_{p, q}^{2} \approx H_{p}\left(X ; h_{q}(\mathrm{pt})\right) \text {. }
$$

An analogous spectral sequence may be defined converging to $h^{*}(X)$ for $h^{*}$ a generalized cohomology theory. Here we have $E_{2}^{p, q} \approx$ $H^{p}\left(X ; h^{q}(\mathrm{pt})\right)$ and if $\operatorname{dim} X=n$, the filtration has the form

$$
0=J^{n+1, q-1} \subseteq J^{n, q} \subseteq \cdots \subseteq J^{0, n+q}=h^{n+q}(X)
$$

where

$$
J^{p, q}=\operatorname{kernel}\left[h^{p+q}(X) \rightarrow h^{p+q}\left(X^{p-1}\right)\right]
$$

and

$$
J^{p, q} / J^{p+1, q-1} \approx E_{\infty}^{p, q} .
$$

2. Products in the spectral sequence. Suppose that we have a slant product $h^{*} \otimes k_{*} \rightarrow h_{*}$ in the sense of $\$ 1$. Let $X$ and $Y$ be finite CW complexes and denote by $\left\{E_{k}^{p, q}\right\},\left\{E_{p, q}^{k}\right\}$ and $\left\{\tilde{E}_{p, q}^{k}\right\}$ the spectral sequences associated with $h^{*}(X), k_{*}(X \times Y)$ and $h_{*}(Y)$, respectively.

Note that

$$
\begin{aligned}
E_{r, s}^{1} & =k_{r+s}\left((X \times Y)^{r},(X \times Y)^{r-1}\right) \\
& \approx \sum_{p} k_{r+s}\left(\left(X^{p}, X^{p-1}\right) \times\left(Y^{r-p}, Y^{r-p-1}\right)\right)
\end{aligned}
$$

and let

$$
\pi_{p}: E_{r, s}^{1} \rightarrow k_{r+s}\left(\left(X^{p}, X^{p-1}\right) \times\left(Y^{r-p}, Y^{r-p-1}\right)\right)
$$

be projection onto the direct summand. Then there is a pairing

$$
\iota_{1}: E_{1}^{p, q} \otimes E_{r, s}^{1} \rightarrow \tilde{E}_{r-p, s-q}^{1}
$$

given by 


$$
a /{ }_{1} b=a / \pi_{p}(b) .
$$

Since for each $n, Z_{n}^{p, q} \subseteq E_{1}^{p, q}$ and $Z_{r, s}^{n} \subseteq E_{r, s}^{1}$, there is a product induced by $l_{1}$ on these subgroups. In order to show that this gives a well-defined product on the $E_{n}$-terms, the following properties must be established:

(i) $Z_{n}^{p, q} / Z_{r, s} \subseteq \tilde{Z}_{r-p, s-q}^{n}$,

(ii) $\left.B_{n}^{p, q}\right|_{1} Z_{r, s}^{n} \subseteq \tilde{B}_{r-p, s-q}^{n}$,

(iii) $Z_{n}^{p, q} / B_{r, s}^{n} \subseteq \tilde{B}_{r-p, s-q}^{n}$.

We will prove (i); the verification of (ii) and (iii) follows from similar arguments. Consider the subcomplexes of $X \times Y$ given by

$$
\begin{aligned}
& A=X^{r} \times Y^{0} \cup X^{r-1} \times Y^{1} \cup \cdots \cup X^{p+k} \times Y^{r-p-k}, \\
& B=X^{p+k-1} \times Y^{r-p-k+1} \cup \cdots \cup X^{p} \times Y^{r-p}, \\
& C=X^{p-1} \times Y^{r-p+1} \cup \cdots \cup X^{0} \times Y^{r} \text { and } D=X^{p+k-1} \times Y^{r-p} .
\end{aligned}
$$

Note that $A \cup B \cup C=(X \times Y)^{r}$. Let

$$
\gamma:\left((X \times Y)^{r},(X \times Y)^{r-k}\right) \rightarrow(A \cup D \cup C, A \cup B)
$$

be the inclusion map of pairs. There is also the inclusion

$$
\epsilon:\left(\left(X^{p+k-1}, X^{p-1}\right) \times\left(Y^{r-p}, Y^{r-p-k}\right)\right) \rightarrow(A \cup D \cup C, A \cup B)
$$

which is an excision map. So define a homomorphism $\beta: k_{r+s}\left((X \times Y)^{r},(X \times Y)^{r-k}\right) \rightarrow k_{r+s}\left(\left(X^{p+k-1}, X^{p-1}\right) \times\left(Y^{r-p}, y^{r-p-k}\right)\right)$ by $\beta=\epsilon_{*}^{-1} \circ \gamma_{*}$.

Consider the diagram

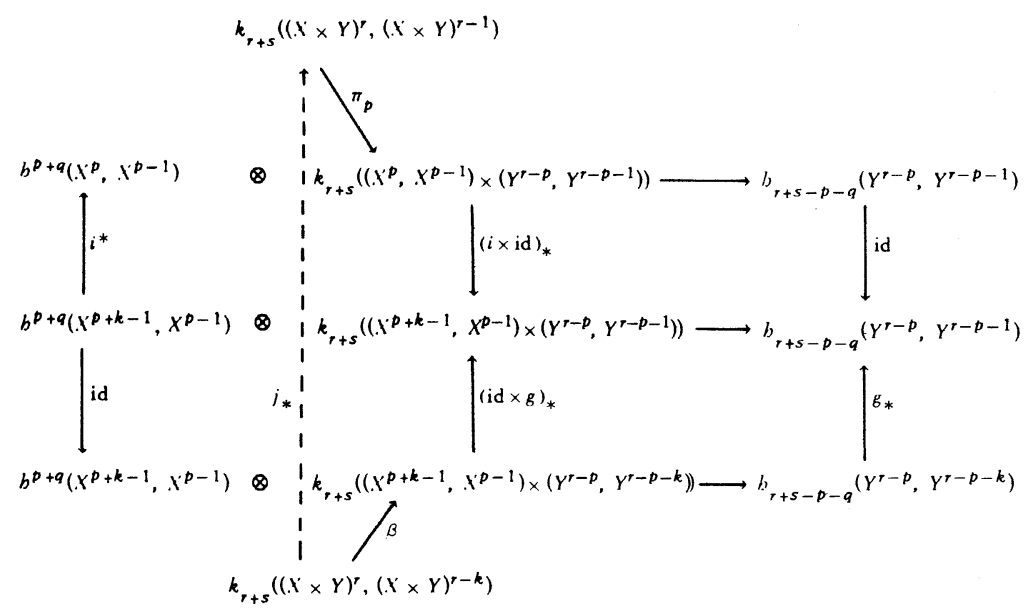


where the maps $i, j$ and $g$ are inclusions. It can be seen from the definition of $\beta$ that the middle loop commutes. Now if $a \in h^{p+q}\left(X^{p+k-1}, X^{p-1}\right)$ and $b \in k_{r+s}\left((X \times Y)^{r},(X \times Y)^{r-k}\right)$ then the functional properties of the slant product imply

$$
\begin{aligned}
i^{*}(a) I_{1} j_{*}(b) & =i^{*}(a) / \pi_{p} j_{*}(b)=a /(i \times \mathrm{id})_{*} \pi_{p} j_{*}(b) \\
& =a /(\mathrm{id} \times g)_{*} \beta(b)=g_{*}(a / \beta(b)) .
\end{aligned}
$$

Thus (image of $\left.i^{*}\right) l_{1}$ (image of $\left.j_{*}\right) \subseteq$ image $g_{*}$ or

$$
Z_{k}^{p, q} / Z_{r, s}^{k} \subseteq \tilde{Z}_{r-p, s-q}^{k} .
$$

This proves (i). The proofs of (ii) and (iii) require only a slight modification of the homomorphism $\boldsymbol{\beta}$ and the diagram (2.1). Therefore, for each integer $m>1$ there is induced a product

$$
l_{m}: E_{m}^{p, q} \otimes E_{r, s}^{m} \rightarrow \tilde{E}_{r-p, s-q}^{m} .
$$

We now want to show that the product given in (2.2) satisfies a derivation formula with respect to the differentials. Let $A \in E_{k}^{p, q}$ and $B \in E_{r, s}^{k}$ be represented by $i^{*}(a)$ and $j_{*}(b)$ respectively, where $i_{*}$ and $j_{*}$ are as in (2.1). Then from the diagram (2.1) we see that $A /{ }_{k} B$ may be represented by

$$
i^{*}(a) l_{1} j_{*}(b)=i^{*}(a) / \pi_{p} j_{*}(b)=g_{*}(a / \beta(b)) .
$$

Then $d^{k}(A / k)$ may be represented by $\partial_{1}(a / \beta(b))$ where

$$
\partial_{1}: h_{r+s-p-q}\left(Y^{r-p}, Y^{r-p-k}\right) \rightarrow h_{r+s-p-q-1}\left(Y^{r-p-k}, Y^{r-p-k-1}\right) .
$$

On the other hand, $d_{k}(A) I_{k} B$ may be represented by $\delta(a) /_{1} j_{*}(b)=$ $\delta(a) / \pi_{p+k} j_{*}(b)$ where

$$
\delta: h^{p+q}\left(X^{p+k-1}, X^{p-1}\right) \rightarrow h^{p+q+1}\left(X^{p+k}, X^{p+k-1}\right) .
$$

Similarly $A I_{k} d^{k}(B)$ may be represented by

$$
i^{*}(a) /_{1} \partial_{2}(b)=i^{*}(a) / \hat{\pi}_{p} \partial_{2}(b),
$$

where

$$
\partial_{2}: k_{r+s}\left((X \times Y)^{r},(X \times Y)^{r-k}\right) \rightarrow k_{r+s-1}\left((X \times Y)^{r-k},(X \times Y)^{r-k-1}\right) .
$$

By applying (1.1), (1.2) and (1.3) these three representatives may be rewritten as follows:

$$
\begin{aligned}
\partial_{1}(a / \beta(b)) & =(-1)^{p+q}\left(a / D_{2} \beta(b)\right), \\
\delta(a) / \pi_{p+k} j_{*}(b) & =a / D_{1} \pi_{p+k}(b),
\end{aligned}
$$




$$
i^{*}(a) / \hat{\pi}_{p} \partial_{2}(b)=a /(i \times \mathrm{id})_{*} \hat{\pi}_{p} \partial_{2}(b) .
$$

Since the slant product is bilinear, it will be sufficient to determine the relationships among the three factors involving $b$. Define subcomplexes of $X \times Y$ by

$$
\begin{array}{ll}
A=X^{p+k} \times Y^{r-p-k}, & C=X^{p+k} \times Y^{r-p-k-1}, \\
B=X^{p+k-1} \times Y^{r-p}, & D=X^{p-1} \times Y^{r-p} .
\end{array}
$$

Duplicating the technique used to define $\beta$, we construct a homomorphsim

$$
\epsilon: k_{r+s}\left((X \times Y)^{r},(X \times Y)^{r-k}\right) \rightarrow k_{r+s}(A \cup B, C \cup(A \cap B) \cup D)
$$

by composing a homomorphism induced by inclusion with the inverse of an excision isomorphism.

Now consider the diagram

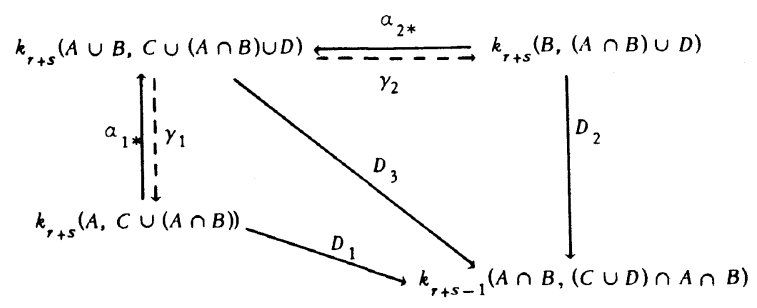

where $\alpha_{1}$ and $\alpha_{2}$ are inclusion maps and $D_{3}$ is defined in the same manner as $D_{1}$ and $D_{2}$, by composing a boundary operator with the inverse of an excision isomorphism. Note that both triangles commute and $\alpha_{1 *}$ and $\alpha_{2 *}$ are monomorphisms onto direct summands. Denoting by $\gamma_{1}$ and $\gamma_{2}$ the corresponding projections, it follows that $D_{3}=D_{1} \gamma_{1}+D_{2} \gamma_{2}$.

A check of the definitions shows that $\gamma_{1}{ }^{\circ} \epsilon=\pi_{p+k}{ }^{\circ} j_{*}$ and $\gamma_{2}{ }^{\circ} \epsilon=\beta$. Finally, the homomorphism $D_{3}{ }^{\circ} \epsilon$ may be factored as the composition $D_{3} \circ \epsilon=(i \times \text { id })_{*}{ }^{\circ} \hat{\pi}_{p} \circ \partial_{2}$. Assembling these facts, we have

$$
(i \times \mathrm{id})_{*} \hat{\pi}_{p} \partial_{2}=D_{1} \pi_{p+k} j_{*}+D_{2} \beta
$$

which when applied to the representatives in (2.3), (2.4) and (2.5) establishes the derivation property

$$
A /_{k} d^{k}(B)=d_{k}(A) l_{k} B+(-1)^{p+q} d^{k}\left(A / l_{k} B\right) .
$$


3. The Poincaré duality thorem. A slant product $h^{*} \otimes k_{*} \rightarrow h_{*}$ is a dual pairing of $h^{*}$ with $h_{*}$ via $k_{*}$ if there exists an element $e \in k_{0}(\mathrm{pt})$ such that the homomorphism $h^{p}(\mathrm{pt}) \rightarrow h_{-p}(\mathrm{pt})$ given by taking $x$ into $x / e$ is an isomorphism for each $p$.

For example let $\mathbf{A}$ be a ring spectrum and $\mathbf{M}$ a spectrum which is an A module (cf. [10]). Then $H^{*}(; \mathbf{M})$ and $H_{*}(; \mathbf{M})$ are dually paired via $H_{*}(; \mathbf{A})$. In particular the sphere spectrum $\mathbf{S}$ is a ring spectrum and the element $e \in H_{0}(\mathrm{pt} ; \mathrm{S})$ is represented by the identity map. Any spectrum $\mathbf{M}$ is a module over $\mathbf{S}$.

Now suppose $h^{*} \otimes k_{*} \rightarrow h_{*}$ is a dual pairing. Let $M$ be a closed $n$-manifold carrying the structure of a finite CW complex. Suppose further that $M$ is orientable for ordinary homology with coefficients in $k_{0}(\mathrm{pt})$. If the spectral sequence $(\$ 1)$ for $k_{*}(M)$ is denoted by $\left\{\hat{E}_{p, q}^{m}\right\}$, this means there is an ordinary fundamental class $\sigma_{2} \in$ $\hat{E}_{n, 0}^{2}$. We define $M$ to be $k_{*}$-orientable if $\sigma_{2}$ is a permanent cycle.

Since $M$ is $n$-dimensional, the inclusion induces a homomorphism

$$
k_{n}(M) \rightarrow k_{n}\left(M^{n}, M^{n-1}\right)
$$

whose image is contained in $\hat{Z}_{n, 0}^{2}=\hat{E}_{n, 0}^{2}$. So it may be interpreted as a homomorphism

$$
\Phi: k_{n}(M) \rightarrow H_{n}\left(M ; k_{0}(\mathrm{pt})\right) .
$$

Then $M$ is $k_{*}$-orientable if and only if $\sigma_{2}$ is in the image of $\Phi$. For example if $k_{*}$ is stable homotopy theory, then $\Phi$ is the stable Hurewicz homomorphism and $M$ is $k_{*}$-orientable if and only if its integral fundamental class is stably spherical.

So let $M$ be $k_{*}$-orientable and fix $\sigma \in k_{n}(M)$ with $\Phi(\sigma)=\sigma_{2}$; $\sigma$ will be called a $k_{*}$-fundamental class. Let $\Delta: M \rightarrow M \times M$ be a skeletal approximation to the diagonal. Then the standard Poincare duality theorem gives an isomorphism $E_{2}{ }^{p, q} \approx \tilde{E}_{n-p,-q}^{2}$ defined by taking $x$ into $x /_{2} \Delta_{*}\left(\sigma_{2}\right)$. Note that $\Delta_{*}\left(\sigma_{2}\right)$ must also be a permanent cycle by naturality. This fact and the derivation property (2.6) of the differentials make it possible to apply standard comparison techniques for spectral sequences and conclude

(3.1) Slanting with $\Delta_{*}\left(\sigma_{2}\right)$ yields an isomorphism

$$
E_{k}^{p, q} \approx \tilde{E}_{n-p,-q}^{k}
$$

for all $p$ and $q$ and for $2 \leqq k \leqq \infty$.

Returning to the filtrations $(\$ 1)$ of the spectral sequences,

(3.2) The homomorphism $h^{k}(M) \rightarrow h_{n-k}(M)$ given by slanting with $\Delta_{*}(\sigma)$ sends $J^{p, k-p}$ into $J_{n-p,-k+p}$. 
To see this, define subcomplexes of $M \times M$ by

$$
\begin{aligned}
& A=M \times M^{n-k} \text { and } \\
& B=M^{k-1} \times M^{n-k+1} \cup M^{k-2} \times M^{n-k+2} \cup \cdots \cup M^{0} \times M^{n} .
\end{aligned}
$$

Note that there is an excision isomorphism

$$
\alpha: k_{n}\left(\left(M, M^{k-1}\right) \times\left(M^{n-k}\right)\right) \stackrel{\approx}{\rightarrow} k_{n}(A \cup B, B) .
$$

Consider the diagram

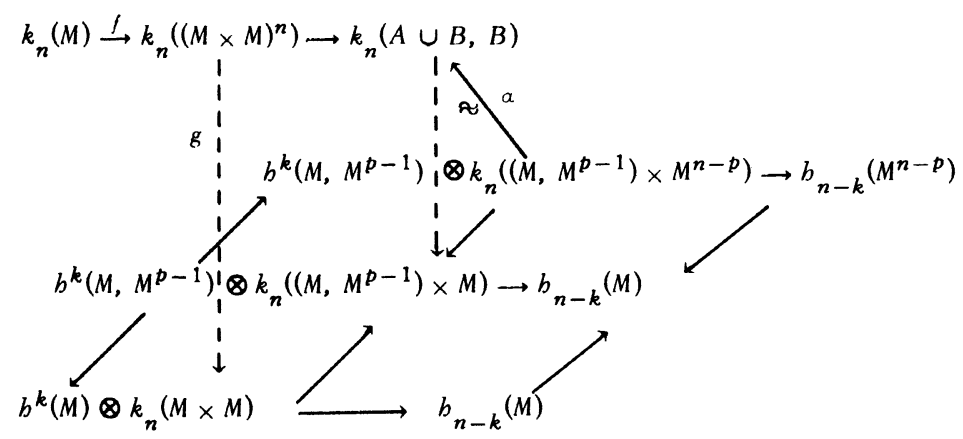

where the composition $g \circ f$ is $\Delta_{*}$ and all the other homomorphisms are induced by inclusions. Then (3.2) follows by diagram chasing.

By combining (3.1) and (3.2) we have

(3.3) The homomorphism $h^{k}(M) \rightarrow h_{n-k}(M)$ given by slanting with $\Delta_{*}(\sigma)$ induces an isomorphism

$$
J^{p, k-p} / J^{p+1, k-p-1} \approx J_{n-p,-k+p} / J_{n-p-1,-k+p+1}
$$

Finally, the fact that $J^{n+1, k-n-1}=0=J_{-1, n-k+1}$ allows us to proceed inductively on the filtrations using (3.2) and (3.3) to complete the proof of the following duality theorem:

(3.4) THEOREM. If $h^{*} \otimes k_{*} \rightarrow h_{*}$ is a dual pairing and $M$ is a closed $n$-manifold which is $k_{*}$-orientable, then there is an isomorphism $h^{k}(M) \approx h_{n-k}(M)$ given by taking $x$ into $x / \Delta_{*}(\sigma)$ where $\sigma \in k_{n}(M)$ is a fundamental class. Moreover, under the natural $\mathrm{CW}$ filtrations, this is an isomorphism of filtered groups.

4. Postnikov factors. Recall that a closed $n$-manifold $M$ is $k_{*}$ orientable if there exists a fundamental class $\sigma_{2} \in \hat{Z}_{n, 0}^{2}=\hat{E}_{n, 0}^{2} \approx$ $H_{n}\left(M ; k_{0}(\mathrm{pt})\right)$ which is a permanent cycle. If it is only known that $\sigma_{2} \in Z_{n, 0}^{m}$, does this mean that $M$ is orientable in some intermediate homology theory? That is, is there a sequence of homology 
theories, proceeding from $H_{*}\left(; k_{0}(\mathrm{pt})\right)$ to $k_{*}$, associated with the successive stages of the spectral sequence in such a way that orientability of $M$ is characterized by whether or not $\sigma_{2} \in \hat{Z}_{n, 0}^{m}$ ? This question has been studied in a different context by Kan and Whitehead [7] for the case $k_{*}=\pi_{*}$, stable homotopy. The relationship with their results will be discussed in $\$ 5$.

The approach we use is due to Dold in [3] and [4] where it was used to study the decomposition of a cohomology theory. If $h_{*}$ is a homology theory and $p$ and $r$ are integers, then for any finite CW complex $X$ define

$$
h_{n}^{(p, p+r]}(X)=\text { Image }\left[h_{n}\left(X^{p+n+r}, X^{p+n-1}\right) \rightarrow h_{n}\left(X^{p+n+r+1}, X^{p+n}\right) .\right.
$$

Note that this group is naturally isomorphic to kernel $\partial^{\prime}$ /image $\partial$ in the composition

$$
\begin{aligned}
h_{n+1}\left(X^{p+n+r+1}, X^{n+n+r}\right) & \stackrel{\partial}{\rightarrow} h_{n}\left(X^{p+n+r}, X^{p+n}\right) \\
& \stackrel{\partial^{\prime}}{\rightarrow} h_{n-1}\left(X^{p+n}, X^{p+n-1}\right),
\end{aligned}
$$

of course the groups will be zero unless $r \geqq 1$. The groups may be defined similarly for any finite CW pair $(X, A)$.

(4.3) $h_{*}{ }^{(p, n+r]}$ is a generalized homology theory.

This follows without difficulty as in [4]. Note that if $f:(X, A) \rightarrow$ $\left(X^{\prime}, A^{\prime}\right)$ is a map of pairs, then $f$ is homotopic to a skeletal map $\bar{f}:(X, A) \rightarrow\left(X^{\prime}, A^{\prime}\right)$ and we define $f_{*}$ to be $f_{*}$. The only property which is not obvious is exactness and this may be established using the characterization in (4.2).

The coefficient group of this homology theory is given by

$$
h_{n}^{(p, p+r]}(\mathrm{pt}) \approx \begin{cases}h_{n}(\mathrm{pt}) & \text { for } p<-n \leqq p+r, \\ 0 & \text { otherwise }\end{cases}
$$

In particular $h_{n}{ }^{(p-1, p]}(X, A) \approx H_{n+p}\left(X, A ; h_{-p}(\mathrm{pt})\right)$.

To describe the relationship between these theories and the spectral sequence for $h_{*}$, let $E_{p, q}^{n}$ be the spectral sequence ( $\left.\$ 1\right)$ for $h_{*}(X)$. There is a filtration

$$
0=F_{0} \subseteq F_{1} \subseteq \cdots \subseteq F_{r}=h_{n}^{(p, p+r]}(X)
$$

where

$$
F_{k}=\text { Image }\left[h_{n}\left(X^{p+n+k}, X^{n+n-1}\right) \rightarrow h_{n}\left(X^{p+n+r+1}, X^{p+n}\right)\right] .
$$

(4.4) In the notation above 


$$
F_{k} / F_{k-1} \approx Z_{p+n+k,-p-k}^{k+1} / B_{p+n+k,-p-k}^{r-k+2} .
$$

To prove this consider the following diagram

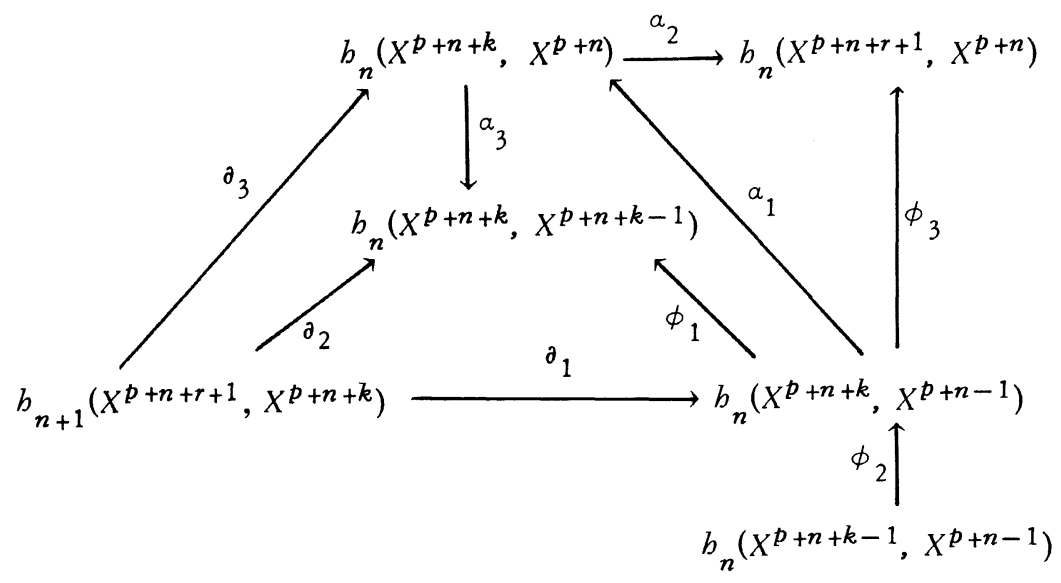

in which $F_{k} / F_{k-1}=$ image $\phi_{3} /$ image $\phi_{3}{ }^{\circ} \phi_{2}$, and image $\phi_{1}=$ $Z_{p+n+k,-p-k}^{k+1}$ and image $\partial_{2}=B_{p+n+k,-p-k}^{r-k-2}$.

Define $\Psi$ : image $\phi_{1} \rightarrow$ image $\phi_{3} /$ image $\phi_{3} \circ \phi_{2}$ as follows: if $\omega=$ $\phi_{1}(x)$, set $\Psi(\omega)$ equal to the coset containing $\phi_{3}(x)$. If $\phi_{1}\left(x^{\prime}\right)=0$, then by exactness there exists a $y$ with $\phi_{2}(y)=x^{\prime}$. But then $\phi_{3}\left(x^{\prime}\right)=$ $\phi_{3} \phi_{2}(y)$ is in the zero coset, so $\Psi$ is well defined. It is evident that $\Psi$ is an epimorphism. By using the diagram, the kernel of $\Psi$ is easily seen to be the image of $\partial_{2}$. This proves (4.4).

The spectral sequence $\left\{{ }^{p, r} E_{s, t}^{k}\right\}$ for $h_{*}^{(p, p+r]}(X)$ is a "truncated" version of the spectral sequence for $h_{*}(X)$ in the following sense:

$$
\begin{aligned}
& { }^{p, r} Z_{s, t}^{k}= \begin{cases}Z_{s, t}^{k} & \text { if } t+p \leqq-k, \\
Z_{s, t}^{-t-p} & \text { if } t+p \geqq-k,\end{cases} \\
& { }^{p, r} B_{s, t}^{k}= \begin{cases}B_{s, t}^{k} & \text { if } t+p+r+1 \geqq k, \\
B_{s, t}^{t+p+r+1} & \text { if } t+p+r+1 \leqq k,\end{cases}
\end{aligned}
$$

so that

$$
p, r Z_{s, t}^{\infty}=Z_{s, t}^{-t-p} \quad \text { and } \quad{ }^{p, r} B_{s, t}^{\infty}=B_{s, t}^{t+p+r+1} .
$$

In this sense, (4.4) says that

$$
F_{k} / F_{k-1} \approx{ }^{p, r} E_{p+n+k,-p-k}^{\infty} .
$$

In a similar fashion, for a cohomology theory $h^{*}$ there are the Post- 
nikov factors $h_{(a, q+r]}^{*}$ which are also cohomology theories. For details see Dold ([3] , [4] ).

In particular

$$
h_{(q-1, q]}^{n}(X, A) \approx H^{q+n}\left(X, A ; h^{-q}(\mathrm{pt})\right) .
$$

There are relations analogous to (4.4) and (4.5) between these theories and the spectral sequence for $h^{*}$.

Now if $h^{*} \otimes k_{*} \rightarrow h_{*}$ is a slant product, the techniques of $\$ 2$ may be applied to show that there is induced a slant product

$$
h_{(q, q+r]}^{*} \otimes k_{*}^{(p, p+r]} \rightarrow h_{*}^{(p-q-1, p+r-q-1]}
$$

for any integers $p, q$ and $r$. If the original product is a dual pairing, the products in (4.6) will also be dual pairings whenever $p<0 \leqq p$ $+r$. Suppose then that this is the case and let $M$ be a closed $n$ manifold with ordinary fundamental class $\sigma_{2} \in H_{n}\left(M ; k_{0}(\mathrm{pt})\right) \approx$ $E_{n, 0}^{2} \approx p, r E_{n, 0}^{2}$. Suppose further that $\sigma_{2}$ remains a cycle until the $k$ th stage, where $k \geqq \max (p+r+1,-p)$. Then since ${ }^{p, r} Z_{n, 0}^{\infty}=$ $Z_{n, 0}^{-p}$ and ${ }^{n, r} B_{n, 0}^{\infty}=B_{n, 0}^{p+r+1}$, we have that $\sigma_{2}$ is a permanent cycle in the spectral sequence for $k_{*}^{[p, p+r]}(M)$. Thus $M$ is $k_{*}^{(p, p+r]}$-orientable and the duality theorem (3.4) may be applied to the pairing (4.6). All of this is summarized in the following:

(4.7) THEOREM. Let $h^{*} \otimes k_{*} \rightarrow h_{*}$ be a dual pairing and $\sigma_{2} \in$ $H_{n}\left(M ; k_{0}(\mathrm{pt})\right) \approx E_{n, 0}^{2}$ be an ordinary fundamental class. Then for $p<0 \leqq p+r$, (4.6) is a dual pairing. If $\sigma_{2} \in Z_{n, 0}^{k}$ where $k \geqq$

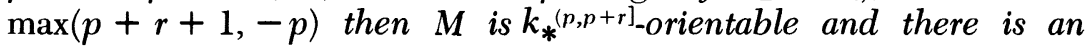
isomorphism

$$
h_{(q, q+r]}^{m}(M) \approx h_{n-m}^{(p-q-1, p+r-q-1]}(M)
$$

of filtered groups for each integer $m$.

5. The results of Kan and Whitehead. The motivating question behind the paper of Kan and Whitehead [7] was whether the gap between the Eilenberg-Mac Lane spectrum $K(Z)$, for which orientability has its usual meaning, and the sphere spectrum $S$, for which a manifold is orientable if and only if it is stably parallelizable, could be filled by interesting spectra and corresponding classes of orientable manifolds. Using semisimplicial methods, they construct a sequence of ring spectra $\left\{A_{i}\right\}_{i=1}^{\infty}$ and maps

$$
K(Z)=A_{1} \stackrel{\phi_{1}}{\leftarrow} A_{2} \stackrel{\phi_{2}}{\leftarrow} A_{3} \stackrel{\phi_{3}}{\leftarrow} \cdots \leftarrow S
$$

so that each $\phi_{i}$ is a fibration of spectra whose fibre is an abelian 
spectrum, i.e. a product of Eilenberg-Mac Lane spectra. Corresponding to the spectrum $A_{n}$, they construct a "universal normal" homology operation (a higher order operation) in ordinary homology and characterize orientability of a manifold for $A_{n}$ by the vanishing of this operation on the ordinary fundamental class. We will view the $A_{i}$ as the topological realizations of the semisimplicial spectra defined in [7].

Let $\pi_{*}$ denote stable homotopy and $\pi_{*}{ }^{(p, p+r]}$ the Postnikov factors in the manner of $\$ 4$.

(5.2) For any $p<0$ and any finite $\mathrm{CW}$ complex $X$ there is a natural isomorphism

$$
\pi_{n}^{(p, 0]}(X) \approx \pi_{n}^{(p, \infty]}(X)
$$

The basic reason for this is that the coefficient group $\pi_{*}(p t)$ is zero in negative dimensions. The proposition may be verified from the diagram

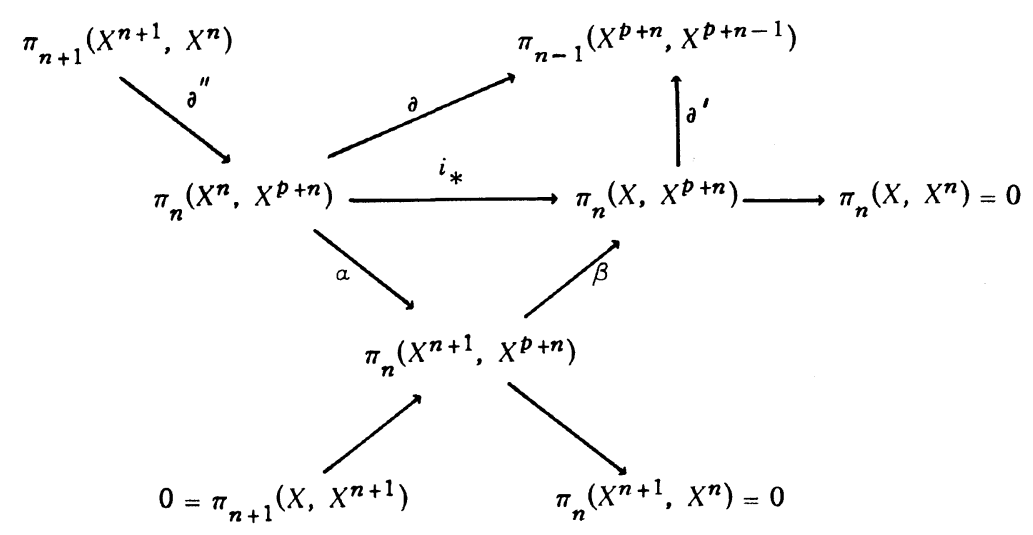

by constructing an isomorphism from

$$
\pi_{n}^{(p, 0]}(X)=\text { kernel } \partial / \text { image } \partial^{\prime \prime} \text { onto } \pi_{n}^{(p, \infty]}(X)=\text { kernel } \partial^{\prime} .
$$

(5.3) For any homology theory $h_{*}$ and integers $p$ and $q$ there is a natural exact triangle

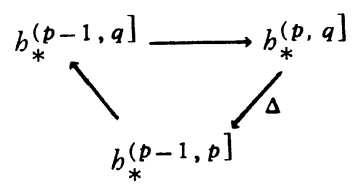

in which $\Delta$ has degree -1 . 
The construction of $\Delta$ from the boundary operator and the verification of (5.3) are easily obtained from the definition (4.1).

Thus there exists a sequence of multiplicative homology theories and natural transformations

$$
H_{*}(; Z)=\pi_{*}^{(-1,0]} \stackrel{\psi_{1}}{\leftarrow} \pi_{*}^{(-2,0]} \stackrel{\psi_{2}}{\longleftarrow} \cdots \leftarrow \pi_{*}
$$

such that each $\psi_{i}$ is part of an exact triangle (5.3) in which the third homology theory is an ordinary homology theory, i.e. it has nonzero coefficient group in only one dimension.

Now by [7, Appendix C] the mapping $S \rightarrow A_{n}$ is an $n$-equivalence. Hence the homomorphism on homology groups

$$
\pi_{*}=H_{*}(; \mathbf{S}) \rightarrow H_{*}\left(; A_{n}\right)
$$

gives a natural transformation

$$
\boldsymbol{\theta}: H_{*}\left(; A_{n}\right) \rightarrow \pi_{*}^{(-n, 0]}
$$

which is an isomorphism on the coefficient groups in dimensions $0 \leqq i<n$.

The technique used to construct the "universal normal" homology operation for $A_{n}$ in [7] from the sequence (5.1) when applied to the sequence (5.4) yields the $n$th differential of the spectral sequence for $\pi_{*}$.

A justification for this alternate approach is that orientability for the theories $\pi_{*}{ }^{(-n, 0]}$ may be given a concise geometrical interpretation. It was shown by Milnor and Spanier [8] that a closed connected oriented smooth manifold $M$ is $\pi_{*}$-orientable if and only if the stable normal sphere bundle has the fibre homotopy type of a product bundle. We now want to generalize this result in terms of the Postnikov factors.

Let $M^{n}$ be a closed connected oriented smoothly triangulated $n$ manifold, $M^{k}$ the $k$-skeleton of $M$. Then the collection of dual cells $\left\{B_{i}\right\}$ gives a decomposition of $M$ as a finite $C W$ complex [11]. Denote the $k$-skeleton of this dual structure by $M_{*}{ }^{k}$. This cellular structure is particularly nice since the closure of an $m$-cell is an $m$-ball. Note then that the $(n-k-1)$-skeleton of $M_{*}$ is the union of the boundaries of the duals of $k$-simplices in $M$. Thus passing to the second barycentric subdivision we have:

(5.5) Let $N$ be the regular neighborhood of the k-skeleton $M^{k}$, $N^{0}=N-\partial N$. Then $M-N^{0}$ is a regular neighborhood of $M_{*}^{n-k-1}$. Note that both of these regular neighborhoods have boundary given by $\partial N$. 
Suppose $M$ is embedded smoothly in high dimensional euclidean space and let $\eta$ be the normal bundle. Then $\eta$ restricts to the normal bundle of $N$. We now have the result of Atiyah [1]

(5.6) The Thom space of $\eta$ restricted to $N, N^{\eta}$, is the S-dual of N/วN.

The techniques of Atiyah for manifolds with boundary [1, (3.1) and (3.2)] may be incorporated in Milnor and Spanier's proof [8, Theorem 1] to yield

(5.7) The normal sphere bundle of $N$ has the fibre homotopy type of a product bundle if and only if there is an S-map $\alpha \in\left\{S^{n}, N\right\}$ such that

$$
\alpha_{*}: H_{n}\left(S^{n}\right) \rightarrow H_{n}(N, \partial N)
$$

is an isomorphism.

Note that the composition

$$
(N, \partial N) \stackrel{i}{\rightarrow}\left(M, M-N^{0}\right) \stackrel{j}{\rightarrow}\left(M, M_{*}^{n-k-1}\right),
$$

where $i$ is an excision map and $j$ is given by retracting $M-N^{0}$ onto $M_{*}^{n-k-1}$, will induce isomorphisms of homology groups. This implies the following corollary.

(5.8) The restriction of the stable normal sphere bundle to the $k$-skeleton of $M$ has the fibre homotopy type of a product bundle if and only if there is an S-map $\gamma$ such that

$$
\gamma_{*}: H_{n}\left(S^{n}\right) \rightarrow H_{n}\left(M, M_{*}^{n-k-1}\right)
$$

is an isomorphism.

Such an S-map $\gamma$ represents an element $\{\gamma\}$ of the stable homotopy group $\pi_{n}\left(M, M_{*}^{n-k-1}\right)$ whose image in

$$
\pi_{n}^{(-k, 0]}(M)=\text { Image }\left[\pi_{n}\left(M, M_{*}^{n-k-1}\right) \rightarrow \pi_{n}\left(M, M_{*}^{n-k}\right)\right]
$$

is a fundamental class. Thus we have

(5.9) $M$ is $\pi_{*}^{(-k, 0]}$ orientable if and only if the restriction of the stable normal sphere bundle to the k-skeleton of $M$ has the fibre homotopy type of a product bundle.

6. Examples. To show that these theories are not vacuous we cite two examples. Following Kan and Whitehead [7], let $\Sigma$ be a wedge of $p$ copies of $S^{r}$ and $f$ a map representing a homotopy class [ $f$ ] in $\pi_{2 r-1}(\Sigma)$. Let $K$ be the space obtained by attaching a $2 r$-cell to $\Sigma$ via $f$. Thus the skeleta of $K$ are given by 


$$
K^{n}= \begin{cases}K & \text { for } n \geqq 2 r \\ \Sigma & \text { for } r \leqq n \leqq 2 r-1 \\ \text { pt } & \text { for } 0 \leqq n \leqq r-1\end{cases}
$$

With a suitable choice of the homotopy class of $f, K$ has the homotopy type of closed manifold [7]. Then $K$ is easily seen to be orientable in the usual sense. Moreover, since $\pi_{*}\left(K^{n}, K^{n-1}\right)=0$ for $r+1 \leqq n \leqq$ $2 r-1$, the fundamental class in $E_{2 r, 0}^{2}$ lies in $Z_{2 r, 0}^{r}$. So $K$ is $\pi_{*}^{(-m, 0]_{-}}$ orientable for $0<m \leqq r$. However, the differential $d^{r}: E_{2 r, 0}^{r} \rightarrow$ $E_{r, r-1}^{r}$ is just the boundary operator from $\pi_{2 r}(K, \Sigma)$ to $\pi_{2 r-1}(\Sigma)$ which takes the ordinary fundamental class into $[f]$. Thus $K$ is not $\pi_{*}^{\{-m, 0]}$-orientable for $m>r$. Therefore, for arbitrarily high values of $m$ there are manifolds which are $\pi_{*}^{(-m, 0]}$-orientable but not $\pi_{*}$ orientable.

As a second example, let $h_{*}$ be a homology theory for which

$$
h_{i}(\mathrm{pt}) \approx \begin{cases}Z_{2} & \text { if } i=0 \text { or } n-1, \\ 0 & \text { otherwise, }\end{cases}
$$

and the differential $d^{n}: E_{p, q}^{n} \rightarrow E_{p-n, q+n-1}^{n}$ is the dual of $\mathrm{Sq}^{n}$. Then any closed manifold will have a fundamental class at the $E^{2-}$ stage, however this class will not be in $\mathrm{Z}^{n+1}$ if the fundamental cohomology class is in the image of $\mathrm{Sq}^{n}$. For example, take the manifold to be $R P(2 n)$. Thus we have a homology theory whose coefficient group is a $Z_{2}$-module, for which some closed manifolds fail to be orientable.

\section{BiBLIOGRAPHY}

1. M. F. Atiyah, Thom complexes, Proc. London Math. Soc. (3) 11 (1961), 291-310. MR 24 \#A1727.

2. M. F. Atiyah and F. Hirzebruch, Vector bundles and homogeneous spaces, Proc. Sympos. Pure Math., vol. 3, Amer. Math. Soc., Providence, R. I., 1961, pp. 7-38. MR 25 \#2617.

3. A. Dold, Halbexakte Homotopiefunktoren, Lecture Notes in Math., no. 12, Springer-Verlag, Berlin and New York, 1966. MR 33 \#6622.

4. $\longrightarrow$ On general cohomology, Chaps. 1-9, Lectures given at the Nordic Summer School in Math. (1968), Matematisk Institut, Aarhus Universitet, Aarhus, 1968. MR 40 \#8045.

5. — Relations between ordinary and extraordinary homology, Colloq. Algebraic Topology, Matematisk Institut, Aarhus Universitet, Aarhus, 1962.

6. W. C. Hsiang and C. T. C. Wall, Orientability of manifolds for generalized homology theories, Trans. Amer. Math. Soc. 118 (1965), 532-359. MR 31 \#1670. 
7. D. M. Kan and G. W. Whitehead, Orientability and Poincaré duality in general homology theories, Topology 3 (1965), 231-270. MR 32 \#8335.

8. J. W. Milnor and E. Spanier, Two remarks on fiber homotopy type, Pacific J. Math. 10 (1960), 585-590. MR 22 \#8524.

9. R. E. Stong, Notes on cobordism theory, Mathematical Notes, Princeton Univ. Press, Princeton, N. J.; Univ. of Tokyo Press, Tokyo, 1968. MR 40 \#2108.

10. G. W. Whitehead, Generalized homology theories, Trans. Amer. Math. Soc. 102 (1962), 227-283. MR 25 \#573.

11. J. F. P. Hudson, Piecewise linear topology, Benjamin, New York, 1969. MR 40 \#2094.

University of TeXas at Austin, Austin, Texas 78712 
